\title{
El cuerpo disciplinado y el ocaso de la libertad: Análisis del hospital psiquiátrico y la escuela en el pensamiento de Michel Foucault.
}

\author{
The disciplined body and the occasion of freedom: Analysis of the \\ psychiatric hospital and the school in the thought of Michel \\ Foucault.
}

Esta obra está bajo una Licencia Creative Commons Atribución 4.0 Internacional. DOI: $10.32870 /$ sincronia.axxiii.n75.5a19

\author{
Gustavo Cristóbal Herrera Urízar ${ }^{1}$ \\ Universidad de Playa Ancha \\ gustavoherreraurizar@hotmail.com \\ (Valparaíso, CHILE)
}

Recibido: 07/09/2018

Revisado: $22 / 10 / 2018$

Aprobado: 07/11/2018

\section{RESUMEN}

En el presente artículo se analizará el concepto de Cuerpo disciplinado, entrando propiamente en el análisis foucaultiano a partir del estudio de la disciplina, sus tecnologías y el poder. De este modo se procederá de manera que sea posible patentar cómo es el que Cuerpo se desempeña bajo una metodología disciplinaria invisible pero aplicada en el área de la educación y la salud. Ya no se trata del Poder entendido en su materialidad como un báculo centralizado, no es la Corona o la espada los

\footnotetext{
${ }^{1}$ Doctor (c) políticas y gestión educativa de la Univeridad de Playa Ancha; Profesor de Filosofía de la Pontificia Universidad Católica de Valparaíso, Chile. Magister en Educación con Mención en Evaluación Educativa en la Pontificia Universidad Católica de Valparaíso, Chile. Docente en el programa en Docencia Universitaria en la Universidad de Chile, Areas de investigación: Filosofía Política y Educación. URÍZAR, Gustavo Herrera. LA EVALUACIÓN DEL CONOCIMIENTO Y SU REPERCUSIÓN EN EL PODER: UN ANÁLISIS DESDE EL PENSAMIENTO DE MICHEL FOUCAULT. LÍMITE Revista Interdisciplinaria de Filosofía y Psicología, 2014, vol. 9, no 29, p. 87-96.

Mail: gustavoherreraurizar@hotmail.com
} 
que simbolizan el poder, sino que éste opera como una microfísica en una relación que se despliega en todas las relaciones, un elemento cualitativo presente en todas las prácticas y conductas humanas, siendo por ende posible docilizar el Cuerpo a raíz de un concepto de Poder disciplinario, utilizando las Instituciones básicas -escuela, hospital y cárcel- como herramientas de control a partir del examen que ejercen y construyen los elementos precisos para el control social y la pérdida de la libertad.

Palabras clave: Cuerpo. Disciplina. Examen. Poder. Libertad.

\section{ABSTRACT}

In the present article the concept of the disciplined body will be analyzed, properly entering Foucauldian analysis from the study of the discipline, its technologies and power. In this way, we will proceed in a way that it is possible to patent how the Body works under an invisible disciplinary methodology applied in the area of education and health. It is no longer about Power understood in its materiality as a centralized staff, it is not the Crown or the sword that symbolizes power, but it operates as a microphysics in a relationship that unfolds in all relationships, a qualitative element present in all human practices and behaviors, being therefore possible to docilitate the Body as a result of a concept of disciplinary power, using the basic Institutions -school, hospital and jail- as control tools from the examination they exercise and construct the precise elements for social control and the loss of freedom.

Keywords: Body. Discipline. Exam. Power. Freedom.

\section{El concepto de Cuerpo, disciplina y poder}

Desde una mirada filosófica e histórica ahora se hace necesario, en post de un avance significativo en torno a lo que el filósofo francés entiende por Cuerpo y su relación con la disciplina, precisar conceptualmente ambas determinaciones: 
La disciplina aumenta las fuerzas del cuerpo (en términos económicos de utilidad) y disminuye esas mismas fuerzas (en términos políticos de obediencia). En una palabra: disocia el poder del cuerpo; de una parte, hace de este poder una "aptitud", una "capacidad" que trata de aumentar, y cambia por otra parte la energía, la potencia que de ello podría resultar, y la convierte en una relación de sujeción estricta económica separa la fuerza y el producto del trabajo, digamos que la coerción disciplinaria establece en el cuerpo el vínculo de coacción entre una aptitud aumentada y una dominación acrecentada (Foucault, 2002, p. 83).

Se infiere que el Cuerpo en la medida que es disciplinado aumenta la producción exigida, además aumenta su capacidad "útil" y disminuye su auto-determinación, pues delega la fuerza de su Cuerpo a la voluntad de un "otro": de esta manera el sujeto se "cosifica", ya que, al perder su capacidad de independencia, el Cuerpo no puede emanciparse. Cuando se disciplina el Cuerpo el alma se hunde en las condiciones sistemáticas reiterativas de un proceso que aniquila la voluntad individual, y el Cuerpo recibe indicaciones para convertirse en un hombre máquina. Cuando hacemos referencia a un hombre máquina nos referimos principalmente al tratamiento que realiza Descartes:

Sabiendo cuántos autómatas o máquinas semovientes puede construir la industria humana, sin emplear sino poquísimas piezas, en comparación de la gran muchedumbre de huesos, músculos, nervios, arterias, venas y demás partes que hay en el cuerpo de un animal, consideren este cuerpo como una máquina que, por ser hecha de manos de Dios, está incomparablemente mejor ordenada y posee movimientos más admirables que ninguna otra de las que puedan inventar los hombres. Y aquí me extendí particularmente, haciendo ver que, si hubiese máquinas tales que tuviesen los órganos y figura exterior de un mono o de otro cualquiera animal, desprovisto de razón, no habría medio alguno que nos permitiera conocer que no son en todo de igual naturaleza que esos animales (1995, p. 34). 
Descartes describe tres formas de entender el Cuerpo y que se dan a entender en el extracto anterior de su texto Discusiones del método. Primero, al asumir al sujeto individual como parte de una "industria" humana, o lo que podríamos llamar sociedad, define además la condición de autómata del ser humano que, como dijimos anteriormente, pasa a ser parte de un conglomerado de sujetos que reciben la misma categoría y ésta es pertenecer a una empresa en común, la cual consiste en participar de una denominación: ser hombre máquina. Lo segundo que establece el cartesiano es la distinción entre el hombre y los animales, que, aunque ambos son Cuerpos máquinas, el hombre al ser creado por Dios merece una categoría superior. Y, en tercer lugar, el hombre posee razón, asunto que es relevante para determinar, también, la diferencia con los demás animales. Bajo estas tres diferencias se establece que el hombre es un Cuerpo máquina y potencialmente disciplinado, debido principalmente a su carácter de máquina semoviente, detalle fundamental que se desarrollará a continuación.

Semoviente es un concepto que deriva del latín movens -entis, que hace alusión a la capacidad de moverse a y por sí mismo, este término también se utiliza dentro de la praxis del derecho como:

Bien muebles por naturaleza. Son las cosas muebles propiamente tales, las que por su esencia misma calzan con la definición legal. Se dividen en semovientes y cosas inanimadas. Son semovientes las cosas corporales muebles que o pueden trasladarse de un lugar a otro moviéndose por sí mismas, como los animales² (Orrego, 2001, s/p).

Descartes en su afirmación asume que el hombre posee la característica de ser un cuerpo semoviente, lo cual deja abierta la posibilidad de poder asumir que un sujeto puede ser un objeto para otros, es decir, que puede ser trasladado o modelado por un "otro" siempre y cuando este "otro" posea un poder y discipline a un sujeto, al igual como se doméstica a un animal, una especie 
de condicionamiento oculto en las relaciones de poder. El disciplinamiento del cuerpo semoviente Foucault lo describe como:

El momento histórico de la disciplina es el momento en que nace un arte del cuerpo humano, que no tiende únicamente al aumento de sus habilidades, ni tampoco a hacer más pesada su sujeción, sino a la formación de un vínculo que, en el mismo mecanismo, lo hace tanto más obediente cuanto más útil, y al revés (2002, p. 83).

El objetivo del disciplinamiento, además de gestionar las potencialidades de un Cuerpo y de mantener una jerarquización de poder, es realizar una conexión o vínculo en las acciones mismas de un sujeto, es decir, que el cuerpo de un sujeto se adecue a las de una estructura rígida que con el paso del tiempo hace de sujeto obediente a las órdenes que se le entregan y útil en su quehacer: obediencia y utilidad se co-pertenecen en la medida que el sujeto se vuelve disciplinado y se homogeneíza a las condiciones que el poder lo requiera. Es decir, es una técnica "centrada en el cuerpo, produce efectos individualizadores, manipula el cuerpo como foco de fuerzas que hay que hacer útiles y dóciles a la vez" (Foucault, 2006, p. 225). Para clarificar el concepto mismo de disciplina, se puede recurrir a la explicación que Judith Revel proporciona de tal:

El régimen de disciplina se caracteriza por cierta cantidad de técnicas de coerción que se ejercen según un control sistemático del tiempo, del espacio y del movimiento de los individuos, e invisten particularmente las actitudes, los gestos, los cuerpos. (....) El discurso de la disciplina es ajeno a la ley, o al de la regla jurídica derivada de la soberanía: ella produce un discurso sobre la regla natural, vale decir, sobre la norma ${ }^{3}(2008$, p. 32).

Esta consideración de Revel permite meditar en torno a lo que Foucault continúa explicando en su texto Vigilar y Castigar sobre la relación entre el margen del Cuerpo y la disciplina:

\footnotetext{
${ }^{3}$ Judith Revel es especialista en filosofía francesa e italiana contemporánea, profesora de la Universidad de Paris I y parte del comité científico del Centro Michel Foucault. Su investigación está centrada en la Biopolítica, en la actualidad también trabaja con Toni Negri, filósofo italiano marxista.
} 
Fórmase entonces una política de las coerciones que constituyen un trabajo sobre el cuerpo, una manipulación calculada de sus elementos, de sus gestos, de sus comportamientos. El cuerpo humano entra en un mecanismo de poder que lo explora, lo desarticula y lo recompone (2002, p. 83).

Foucault expresa que el Cuerpo es manipulado por políticas de las "coerciones" las cuales moldean todas las acciones de un sujeto (gestos y comportamientos). Las políticas de las coerciones no pretenden prohibir acciones, sino por el contrario provocarlas, es decir, el poder no determina leyes que no deben ser violadas, sino normas que deben ser ejecutadas, más adelante se precisará con mayor exactitud como aborda el filósofo francés la diferencia entre ley y norma. Ahora bien, volviendo al tema del Cuerpo manipulado, es que la disciplina reconoce al sujeto explorándolo, definiéndolo situadamente en un contexto, lo cual podría definirse como que el cuerpo es observado minuciosamente, luego es desarticulado, es decir, divide las potencialidades que pueda ejecutar y a partir de esto lo reestructura o recompone, ya que el mecanismo de poder que lo reconoció y desarticuló lo vuelve a armar, curiosamente como una máquina en mal estado, reacondicionada y nuevamente operativa, pero con los intereses que requiera el mecanismo de poder. Una de las características de la disciplina es que "trata de regir la multiplicidad de los hombres en la medida en que esa multiplicidad puede y debe resolverse en cuerpos individuales que hay que vigilar, adiestrar, utilizar y, eventualmente, castigar" (Foucault, 2006, p. 220) por ende, se trata de un poder que se dirige y puntualiza en cada cuerpo particular, es el Cuerpo el foco del Poder.

En relación con el estudio de la disciplina como modo de dominación, de acuerdo a la revisión que realiza Foucault (2002), ésta surge como tal a fines del siglo XVIII, comienzos del siglo 
$\mathrm{XIX}^{4}$, y hace referencia de forma explícita a la producción de una anatomía política del cuerpo o a una mecánica del poder desde.

Una 'anatomía política', que es igualmente una 'mecánica del poder', está naciendo; define cómo se puede hacer presa en el cuerpo de los demás, no simplemente para que ellos hagan lo que se desea, sino para que operen como se quiere, con las técnicas, según la rapidez y la eficacia que se determina (Foucault, 2002, p. 83).

La mecánica del poder está determinando una relación de poderes, es decir, hay un juego de poder o choque posesivo de intenciones en las cuales un sujeto recibe indicaciones para sus acciones, una forma de normar las conductas humanas en todos los aspectos vitales, y principalmente, productivos; entender el Poder es comprehender su carácter dominador a partir de las estrategias de las instituciones, el poder es una relación transversal a todos los humanos y toda relación se constituye como relación de poder. Pero antes de seguir adelante con la relación Cuerpo y disciplina es necesario detenerse en lo que entiende Foucault por el concepto de poder, debido principalmente, a que es el mecanismo de poder lo que hace posible el nexo entre Cuerpo y disciplina.

(...) la palabra 'poder' amenaza introducir varios malentendidos. Malentendidos acerca de su identidad, su forma, su unidad. Por poder no quiero decir 'el Poder', como conjunto de instituciones y aparatos que garantizan la sujeción de los ciudadanos en un estado determinado. Tampoco indico un modo de sujeción que, por oposición a la violencia, tendría la forma de la regla. Finalmente, no entiendo por poder un sistema general de dominación ejercida por un elemento o un grupo sobre otro, y cuyos efectos, merced a

\footnotetext{
${ }^{4}$ Foucault al analizar el régimen disciplinario en esta época histórica, no limita su estudio solamente a tal período, sino que le sirve como antesala al estudio de la nueva forma de organización del poder: la biopolítica, que, en sus palabras y para distinguirla del régimen disciplinario-: "La nueva tecnología introducida está destinada a la multiplicidad de los hombres, pero no en cuanto se resumen en cuerpos sino en la medida en que forma, al contrario, una masa global, afectada por procesos de conjunto que son propios de la vida, como el nacimiento, la muerte, producción, la enfermedad, etc. Por lo tanto, tras un primer ejercicio del poder sobre el cuerpo que se produce en el modo de la individualización, tenemos un segundo ejercicio que no es individualizador sino masificador, por decirlo así, que no se dirige al hombre/cuerpo sino al hombre/especie" (Foucault, 2006, p.220.)
} 
sucesivas derivaciones, atravesarían el cuerpo social entero. El análisis en términos de poder no debe postular, como datos iniciales, la soberanía del estado, la forma de la ley o la unidad global de una dominación; éstas son más bien formas terminales. Me parece que por poder hay que comprender, primero, la multiplicidad de las relaciones de fuerza inmanentes y propias del dominio en que se ejercen, y que son constitutivas de su organización; el juego que por medio de luchas y enfrentamientos incesantes las trasforma, las refuerza, las invierte; los apoyos que dichas relaciones de fuerza encuentran las unas en las otras, de modo que formen cadena o sistema, o, al contrario, los corrimientos, las contradicciones que aíslan a unas de otras; las estrategias, por último, que las tornan efectivas, y cuyo dibujo general o cristalización institucional toma forma en los aparatos estatales, en la formulación de la ley, en las hegemonías sociales (Foucault, 1977, p. 55).

Cabe mencionar que Foucault prioriza el proceso por el fin, ya que lo que el autor pretende es develar los mecanismos ocultos por las relaciones humanas: la relación de un sujeto con otro genera en el acto mismo una lucha y enfrentamiento de intereses por la propia estructura conceptual que posee, lo que gatilla en una red o sistema de reproducciones simbólicas que se vuelven efectivas al ser institucionalizadas. En otras palabras, lo que nos dice Foucault es que los seres humanos en sus relaciones generan sociedad y el Cuerpo disciplinado no es más que el producto de estas relaciones, donde hay una lucha constante en el diálogo mismo y que es delimitado, posteriormente, por las instituciones, supliendo el debate y determinando a un sujeto al quehacer definido por ellas (las instituciones). "El poder no es una institución, y no es una estructura, no es cierta potencia de la que algunos estarían dotados: es el nombre que se presta a una situación estratégica compleja en una sociedad dada." (Foucault, 1986, p. 113)

El poder para Foucault no es dado, no es innato, no pertenece a alguien específico, ni es una red o sistema rígido, el poder está presente en todos los hombres y se hace patente en las relaciones. Pero se debe recalcar lo que se mencionó anteriormente, y es que no es lo mismo "el 
poder" que "el Poder": recordemos que por poder no quiere decir "el Poder", como conjunto de instituciones y aparatos que garantizan la sujeción de los ciudadanos en un estado determinado. El Poder hace alusión a la concepción aún moderna en tanto entendido como Poder centralizado y concentrado en las instituciones; el poder es en cambio el despliegue de relaciones, pues en toda relación se materializa una relación de poder. Es necesaria esta distinción ya que "el poder" y "el Poder" son utilizados distintamente en esta investigación, asunto medular en el análisis en lo expresado por el autor.

El poder al que hace referencia Foucault está presente en diversas acciones mediatizadas en la mayoría de las veces por instituciones que no valoramos en la medida necesaria, esto se expresa cuando analizamos la labor de la economía en nuestras vidas. Trabajamos y realizamos labores sin analizar el por qué y el para qué de esto. Si reflexionamos a fondo la labor que se realiza cuando se trabaja, se llega a la siguiente conclusión: que el porqué deviene de la disciplina oculta de nuestras acciones. "Si la explotación económica separa la fuerza y el producto del trabajo, digamos que la coerción disciplinaria establece en el cuerpo el vínculo de coacción entre una aptitud aumentada y una dominación acrecentada" (Foucault, 2002, p. 83).

Siguiendo una lógica de relaciones causales, podríamos decir que la fuerza es equivalente a la aptitud aumentada o de mejor capacitación para un fin u objetivo, y el producto del trabajo es equivalente a una dominación acrecentada, es decir, al ver un "buen" trabajo el sujeto asume que es "bueno" en lo que hace y se siente parte de un proceso productivo. En otras palabras, la mediación de la disciplina permite que el sujeto se someta a un proceso, el cual potencia lo necesario para que el sujeto sea cada vez más apto en su desempeño, y al ver un resultado positivo legitima el proceso y se siente parte de él. De esta manera el sujeto no cuestiona su desempeño laboral o trabajo y la plusvalía de su trabajo no es analizada. En este sentido la explotación económica que menciona Foucault deja entrever que lo que pretende el autor en su dimensión de análisis es apreciar que el sujeto en la medida que se encuentre arraigado en un modelo de producción que este sustentado en instituciones, sean estas empresas comerciales o el mismo estado, el sujeto pierde su 
independencia y delega su poder, muchas veces, sin tener conciencia del juego cuerpo-disciplina presente en las instituciones. Sin embargo, cabe precisar que el análisis foucaultiano responde necesariamente al análisis de la sociedad del siglo XVIII- XIX, y por ende, la relación que se establece entre disciplina, trabajo y producción, no apunta a un análisis del capitalismo.

el sistema de poder, bajo su mecanismo disciplinario, no es una consecuencia del modelo capitalista de producción, sino un elemento constitutivo e inmanente que hace posible el funcionamiento de la sociedad industrial (Castro, 2008, p. 151).

Esta referencia a la independencia del sujeto y la delegación de poder que se atribuye al análisis de la disciplina que hace Foucault tiene que ver con la influencia filosófica de Heidegger en el pensamiento de Foucault, esto se expresa fundamentalmente en la importancia de ver al sujeto en su estado de existencia prima, en el estar ahí, en el Dasein que aborda Heidegger, el cual puede ser extrapolado conceptualmente -guardando las precisiones histórico espaciales- al poder de Foucault. Lo que agrega Foucault a la existencia es su capacidad de situarse y verse a sí mismo con el fin de cambiar, asumir su tiempo y analizar lo que hace.

\section{Tecnología de la Disciplina}

Michel Foucault establece en el tercer capítulo "Disciplina" de Vigilar y Castigar, específicamente en el subtítulo "Los medios del buen encauzamiento", la tecnología de la disciplina: "El individuo es sin duda el átomo ficticio de una representación 'ideológica' de la sociedad; pero es también una realidad fabricada por esa tecnología específica de poder que se llama la 'disciplina'." (Foucault, 2002, p. 118). La tecnología que hace referencia Foucault es un concepto que es necesario revisar con detención, ya que no se sostiene como la tecnología científica que estamos acostumbrados a poseer en los aparatos que utilizamos a diario, sino que apunta más bien al análisis de las prácticas del sujeto, es decir, cómo es que el sujeto convive con ciertas acciones que lo determinan, de esta manera es que estas prácticas se definen: 
Por la regularidad y la racionalidad que acompañan los modos de hacer. Esta regularidad y esta racionalidad tienen, por otro lado, un carácter reflejo; son objeto de reflexión y análisis. Los términos 'técnica' y 'tecnología' agregan a la idea de práctica los conceptos de estrategia y táctica. En efecto, estudiar las prácticas como técnicas o tecnología consiste en situarlas en un campo que se define por la relación entre medios (tácticas) y fines (estrategia). Foucault ha utilizado esta terminología y esta conceptualización, en primer lugar, para establecer una metodología del análisis del poder (la disciplina y el biopoder) (Castro, 2004, p. 524).

De acuerdo a lo mencionado anteriormente es que cuando se habla de tecnología de la disciplina a lo que se hace referencia es al análisis del poder de la disciplina que repercute en los quehaceres o acciones de un sujeto. Este análisis del poder es sobre el Cuerpo que es instrumentalizado de acuerdo a los mecanismos que determine el poseedor del poder sobre el Cuerpo, de esta forma es que esta instrumentalización no repercute en el sujeto esporádicamente o no situadamente, sino que es cotidiana y en todo momento. Es un poder constante, cronológico y espacial que determina al sujeto. El Cuerpo que es disciplinado se establece como un único Cuerpo que debe ser generalizado, o en otros términos, estandarizado. La "construcción" de un cuerpo, es decir, el ideal que debe ser un sujeto se realiza por intermedio de la disciplina, es una "construcción" de las acciones de un sujeto que a posteriori determinará lo que será el sujeto.

Anteriormente se ha mencionado que la disciplina se realiza sobre un sujeto que es el ideal y que posteriormente será generalizado, es decir se pretende determinar un sujeto que será igual en muchos otros sujetos, es por ello que Foucault sostiene que: "Las disciplinas marcan el momento en que se efectúa lo que se podría llamar la inversión del eje político de la individualización" (Foucault, 2002, p. 117).

La individualización del sujeto, por un lado, asume que el Cuerpo puede ser manipulado y por otro lado, y aún más importante, que un sujeto es definido según las acciones que cumple al igual que otros que comparten la misma disciplina en sus oficios. 
En un régimen disciplinario, la individualización es en cambio 'descendente': a medida que el poder se vuelve más anónimo y más funcional, aquellos sobre los que se ejerce tienden a estar maś fuertemente individualizados; y por vigilancias más que por ceremonias, por observaciones más que por relatos conmemorativos, por medidas comparativas que tienen la 'Norma' por referencia, y no por genealogías que dan los antepasados como puntos de mira; por 'desviaciones' más que por hechos señalados (Foucault, 2002, p. 118).

El cuerpo ya no debe ser castigado ni asesinado en la vía pública para servir como ejemplo a lo que no se debe hacer, el miedo no pretende ser visible como antaño, ahora en cambio, para determinar a un sujeto lo que se realiza es una disciplina de las acciones de un sujeto. El que posee un poder no pretende ser visibilizado directamente, sino que las acciones son el poder mismo de, valga la redundancia, un poderoso (cuando se habla de poderoso no se habla desde una perspectiva de un sujeto que sea más o mejor que otro, sino el que detenta la autoridad). Bajo lo definido por Foucault en el texto anteriormente señalado, es que se hace notorio dos puntos que son muy relevantes en su obra Vigilar y castigar, primero es su análisis sobre la vigilancia a la disciplina individualizada y en segundo lugar, la norma que debe regir para demarcar lo que puede o no hacer un sujeto. En ambas situaciones, se apela a determinar lo que se debe hacer, aniquilando la libertad individual de un sujeto y situando en su labor la responsabilidad social, es decir, mientras mejor realiza una labor mejor es el aporte social.

Las acciones que se realizan en una labor son minuciosamente observadas para mantener la norma, con el fin de que se cumpla lo que se pretende en un sistema total y absolutamente estructurado. Por lo tanto, la norma de antemano define lo que es bueno, no hay azar en esto, y no da pie a lo malo, a lo equívoco o lo incorrecto. De acuerdo a lo anterior es que la disciplina lo que pretende es:

(A través de una infrapenalidad otra letra que no es ley sino norma, diferencia que veremos más adelante) sancionar todo aquello que no se ajusta a la regla, reduciendo la 
posibilidad de desviación o la diferencia. Dicha sanción se realiza refiriendo los actos individuales a una norma que sirve de comparación, midiendo términos cuantitativos y jerarquizando en términos de valor las capacidades de los individuos o trazando el límite que habrá de definir la diferencia y la frontera exterior de lo normal (Castro, 2004, p. 524).

La homogeneización de los sujetos y la normalización determinada es lo que caracteriza la disciplina y es ésta la que constituye la tecnología de la que dispone; en este sentido el término norma es muy importante en el análisis de la disciplina que realiza Foucault y esto se debe principalmente a que se establece una relación entre disciplina y poder.

Foucault sostiene que para abordar la cuestión del poder es necesario dejar de lado los conceptos tradicionales de 'ley' o 'soberanía', así como también la noción de represión, que ofrece una representación sólo negativa de sus mecanismos. Para Foucault, en su forma moderna el poder se ejerce cada vez más en un dominio que no es el de la ley, sino el de la norma, y, por otro lado, no simplemente reprime una individualidad o una naturaleza ya dada, sino que positivamente la constituye, la forma. Foucault distingue dos modalidades fundamentales de ejercicio del poder en las sociedades occidentales y modernas: la disciplina y la biopolítica, es decir, el poder que tiene como objetivo los individuos y el poder que se ejerce sobre las poblaciones (Castro, 2004, p. 524).

En este sentido, y en relación al Cuerpo, podemos aseverar que a partir del concepto de norma comparecen las dos formas de dominación sobre tales, en el régimen disciplinario el poder se ejerce individualmente sobre los cuerpos bajo la conducción y ritualización de tales; en la biopolítica, no se trata ya de ejercer individualizadamente el poder, sino de abarcar la población y con ello, atacar a los Cuerpos mediante códigos polimórficos y transversales a la sociedad y a la vida.

Ahora bien, cuando Foucault sostiene la necesidad de distinguir entre ley y norma, lo que hace es situar el poder que se ejerce sobre un sujeto en la medida concreta en que se desenvuelve la disciplina, es decir -e invirtiendo lo anteriormente dicho-, es como la disciplina se hace patente en 
los sujetos cuando se hace referencia a la norma que introduce el que posee un poder sobre uno o más sujetos, es por ello que Foucault establece que la ley está determinada para establecer la corrección de algo que debe ser prohibido y con ello reprimir una acción, pero la norma es algo muy distinto, por lo cual será revisada a continuación.

El autor establece, de acuerdo a aquello que ya se ha mencionado anteriormente, que la tecnología de la disciplina consiste en la relación entre el poder y el disciplinamiento de un sujeto, ahora bien, esta disciplina está normada, esto quiere decir que existen cánones, pautas, estandarizaciones, etc., cualquiera de estos nombres servirá en este caso para decir que lo que se pretende es calificar lo que se debe realizar, y a diferencia de la ley que pretende sancionar lo que no se debe hacer (representación negativa del quehacer de un mecanismo de justicia). La norma estructura al sujeto, moldea los intereses y acciones que debe cometer. Para ser más precisos respecto al concepto de norma: "las disciplinas son ajenas al discurso jurídico de la ley, de la regla entendida como efecto de la voluntad soberana. Por el contrario, la regla disciplinaria es una regla natural: la norma" (Revel, 2008, p. 62). En este sentido, con la norma Foucault proclama la aparición de un biopoder que se instaura a partir de una "medicina social" que se posiciona en los siglos XVII y XVIII para intervenir los aspectos de la vida de los hombres. Ahora bien, y de acuerdo a lo anteriormente expresado, se hace evidente que la disciplina:

No opera a través de la ley, sino que actúan en sus intersticios. La ley no tiene potestad en la tecnología de sujeción de los cuerpos. Esta tarea recae en un 'sistema de no-derecho' que se sirve de la norma como patrón de ordenamiento, control y elaboración de la población. En las instituciones disciplinarias y en el resto de la sociedad, hay que distinguir el universo de las libertades que proclama la teoría jurídico-liberal con el imperio de la ley, del universo paralelo de una infrapenalidad orientada al sometimiento de los individuos (Castro, 2008, p. 152).

En tal sentido, afirma Foucault (2002): "Las disciplinas reales y corporales han constituido el subsuelo de las libertades formales y jurídicas" (p. 134). La norma determina el control del cuerpo, 
ya que como se dijo anteriormente, ordena y elabora la población, es por ello que lo que realiza Foucault en su análisis es re-estructurar lo que se entiende por obligatoriedad de realizar una acción, ya que por un lado está la teoría jurídica que trata el cómo se coartan las libertades individuales en una población (deberes y derechos) y por otro lado, se encuentra un ordenamiento implícito que subyuga al sujeto a realizar acciones que no están amparadas en la ley. Para dejar claro, no se pretende abordar la ilegalidad de las acciones, sino más bien, lo que se pretende establecer es que la norma sitúa al cuerpo en su quehacer, en la repetición sistemática y localización de las laborares. Existe un lenguaje oculto en la relación entre el que posee poder y el que acata al poderoso, asunto que se devela en el análisis de las instituciones que son las que diseñan las relaciones humanas. Lo que pretende la norma para el sujeto no es coartar la individualidad sino fabricarla y para ello la tecnología de la disciplina se sitúa espacialmente vinculada a un conjunto de instituciones en las que son ejecutadas.

El resultado de un proceso complejo que se lleva a cabo con el respaldo de una red de instituciones que se sirven de esta tecnología para sus fines. Tales instancias son: la prisión, la escuela, el taller o el cuartel militar (Castro, 2008, p. 149).

El análisis de las instituciones que realiza el filósofo francés está relacionado con la disciplina que proyectan para con los individuos que se sienten responsables en asistir a ellas. El asunto presente, y que es necesario aclarar, es el por qué de la necesidad de la asistencia o presencia de las personas a las instituciones disciplinarias. Esto se debe básicamente a un factor fundamental que es la relevancia que posee el estado en el quehacer de los hombres, sobre todo en su ordenamiento jurídico que estructura las leyes de una sociedad:

No ha cesado de trabajar en profundidad las estructuras jurídicas de la sociedad para 'hacer funcionar los mecanismos efectivos del poder en oposición a los marcos formales que se había procurado. Las Luces, que han descubierto las libertades, inventaron también las disciplinas (Foucault, 2002, p. 134). 
La profundidad del análisis de Foucault procura una reflexión independiente de cánones o prejuicios. Se puede apreciar por tanto una "observación intelectual" que devela la realidad de un tema que muchas veces se oculta por parte de los que defienden el poder para mantener un status quo social. Por un lado, que el ordenamiento jurídico de un estado entrega libertades (derechos) y que esta misma se sustenta por disciplinas que determinan a priori que el quehacer de un sujeto está limitado por lo dado, por ese derecho y por la disciplina que moldea el cómo se desarrolla el cumplimiento de la libertad.

En otras palabras, lo que la libertad establece en un ordenamiento jurídico es que promueve el "amoldamiento" de un sujeto a una realidad definida de antemano, que no es más que la decisión de unos pocos sujetos y replicados en las instituciones para disciplinar a la sociedad en su conjunto. La libertad no es más que la aceptación y adopción de un ordenamiento jurídico basado en la disciplina del poder en una institución determinada. Anteriormente se ha expresado la relación presente entre el cuerpo y la disciplina, ahora bien, es necesario determinar dónde se desarrolla la disciplina del cuerpo y esto es en una institución.

A este registro de orden espacial que contempla la tecnología del poder alojado en una institución también considera una forma de distribución y mecanismo de relaciones de poder en un tiempo determinado. En este sentido:

Determinadas Instituciones de poder como el hospital, la prisión o la escuela han requerido de prácticas y discursos operativos para hacer efectiva la producción de individuos disciplinados. Es en este contexto y dada tal exigencia de saber, donde se han desarrollado evidencias y reglas sobre las cuales se han edificado las ciencias humanas (Castro, 2008, p. 153).

Las Instituciones nacen de la necesidad de generar un sujeto tipo por parte del poder, este sujeto acude a las instituciones por leyes que regulan el accionar cotidiano de las personas y pretenden normar al sujeto, además se utilizan discursos o relatos que fomentan lo necesario y hasta impostergable de asistir a instituciones que diseñen el marco teórico y práctico de un sujeto, por 
ejemplo, asistir a una escuela o acceder a un centro asistencial de salud, asunto que se verá con mayor detención más adelante.

Se puede adelantar eso sí, en decir que las Instituciones establecen a priori el qué de su función, se nace sabiendo que existen y no requieren análisis sobre su utilidad, se supone su asistencia y se consolidan como parte de un proceso normal en el desarrollo de las ciencias, como si fuesen parte de nuestro contexto familiar, las instituciones son parte de nuestras vidas. Pero desde esta misma perspectiva se puede apreciar que las instituciones disciplinarias: "Han brindado un verdadero laboratorio para el desarrollo de un conocimiento del hombre en cuanto objeto" (Castro, 2008, p. 153), lo curioso es que los mismos sujetos que son obligados por ley (obligatoriedad) o relato para asistir a una Institución, también son observados para definir el futuro de las mismas y el cómo de sus procesos para seguir manteniendo la institucionalidad. Esto quiere decir que aunque los sujetos tengan ciertas características que pretendan ser singulares, estas mismas están siendo observadas para ser introducidas en el proceso institucional. En este sentido, y respecto al Cuerpo, éste es también normado bajo códices y disciplinamientos, el Cuerpo en tanto elemento constituyente del individuo también es institucionalizado e incluso, en tanto que tal, ritualizado mediante las prácticas carcelarias, de salud y escolares -modos de existencia de las principales instituciones- que doblegan su actividad a una repetición cultual en cuanto Cuerpo útil y trabajador, en definitiva, un Cuerpo dócil y disciplinado.

Todo suma en la medida que se pretenda mantener un status quo de un sistema "normalizador" de las personas a través del disciplinamiento de sus cuerpos. Esto significa irremediablemente que existen ciertos patrones institucionales para aplicar, mantenerse y control hasta el día de hoy este displinamiento: "Los espacios institucionales mencionados (cárcel, hospital, escuela) como las ciencias del hombre poseen una matriz histórica en común que es el poder disciplinario" (Huber, 1968, p. 180).

A continuación, es necesario analizar brevemente las tres instituciones que son parte de la revisión teórica que realiza Michel Foucault en su texto Vigilar y Castigar, para dar cuenta cómo 
opera este mecanismo de poder o anatomía política en los cuerpos. Las instituciones que Foucault analiza, y que ya han sido mencionadas anteriormente, son: la cárcel (prisión), el hospital y la escuela, patentizando con esto la visión de panóptico en cuanto tales instituciones operan como mecanismo de control en tanto existe una vigilancia constante en busca de la corrección de hábitos y conductas; esta lectura además permite contextualizar el análisis foucaultiano en el ámbito contemporáneo, a casi cuarenta años de su redacción.

La cárcel en su praxis se expresa a través de la prisión de un sujeto. La prisión pretende tomar al individuo que infringe la ley, no para capturar su cuerpo y que éste tome conciencia del crimen que ha cometido -el cual es definido como tal por el estado de un país-, sino para tomar posesión del individuo para disciplinarlo:

No se trata de un artefacto de privación de la libertad que aflore como resultado de una larga búsqueda por parte del estado de derecho, sino que nace como una reorganización de micropoderes presentes con antelación en diferentes capas de la sociedad.(...) en efecto, los cuerpos son excluidos de la vida social para ser integrados en un dispositivo de encierro que, luego de someterlos a una serie de mecanismos (vigilancia, sanción normalizadora y examen), los libera con el propósito de volver más tarde a capturarlos nuevamente en su red disciplinaria. De esa manera, la prisión cumple un rol positivo para la sociedad capitalista; realiza una tarea provechosa desde la perspectiva de la legitimación que exige el sistema imperante (Castro, 2008, p. 153).

La prisión de un sujeto, aunque sea por un hecho que socialmente debiese ser penalizado, debe ser reconsiderada. Primero, porque la penalización exigida ha existido como construcción de un tejido social que exige algo por algo, es decir, a partir de una acción debe haber un resultado, causa y efecto. Esto sucede con una acción jurídicamente prohibida: el hombre que comete una acción debe ser penalizado.

En segundo lugar, al determinar una acción como penalizable afloran las concepciones éticas y morales arraigadas por la cultura judeo-cristianas que las sociedades conservan en su mayoría y 
que el sistema político sostiene, a pesar de los años de evidencia científica; en tercer lugar, la cárcel está determinada para sujetos que reciben por parte del sistema jurídico de un estado una categoría pública negativa, que no es expuesta en la plaza pública como sucedía en el siglo XVII, sino que es archivada en registros de acceso público; estos registros y las categorías en que son penalizados los sujetos son creados por políticos ${ }^{5}$.

El sistema carcelario que se sustenta en la determinación en como entienden la justicia por parte de los políticos es una "bandera de lucha" de estas personas, es así que en la medida en que se acaben los delitos, menor será su eficacia en el sistema, por lo tanto, los políticos necesitan que el sistema carcelario sea un fracaso, ya que de esta manera se justifica su representación. Es así como el fracaso de la prisión se expresa como:

No reformar a los delincuentes, e incluso fomentar su eventual peligrosidad, no constituye una consecuencia indeseada para esta tecnología. Al contrario, el fracaso es inherente a la prisión. Lo que explica que esta institución sea prácticamente contemporánea del discurso y la política de reforma carcelaria (Castro, 2008, p. 154).

La segunda institución que analizáremos según la perspectiva de Michel Foucault es la institución del hospital, que se expresa en la acción de un médico y su relación con un paciente. El filósofo francés en su texto Vigilar y Castigar, exactamente en el apartado tres del subcapítulo dos, del capítulo dos: "el examen" de su obra, expresa el cambio histórico que ha vivido la concepción de médico en la historia francesa. Nos cuenta el paso que ha desarrollado el médico, sobre todo en la jerarquía social en la cual se ubica el sujeto encargado de atender a personas "enfermas". La jerarquía antes mencionada se sitúa en el hospital, la institución que centra su atención a pacientes que son "examinados" por médicos: "Una de las condiciones esenciales para el desbloqueo

\footnotetext{
${ }^{5}$ De este modo podemos considerar que para Foucault todo preso es tal en virtud de motivos políticos: los anormales y marginados -por conductas erráticas o no adecuadas para la sociedad- son encarcelados para corregir tales conductas para hacerlos Cuerpos no sólo dóciles, sino productivos.
} 
epistemológico de la medicina a fines del siglo XVIII fue la organización del hospital como aparato de examinar. El ritual de la visita es su forma más llamativa" (Foucault, 2002, p. 113).

Foucault hace una revisión histórica con información obtenida desde 1661 hasta 1771. El fin era conocer el hilo conductor del proceso histórico del hospital y las funciones del médico presente en ella. Los resultados de esta observación son: primero, que el médico tiene cada vez mayor presencia en la observación de los pacientes, es decir, "visita" al paciente más seguido en una constante examinación; segundo, el médico posee mayor relevancia social que el sacerdote, quitándole importancia a la iglesia. Tercero, el médico posee mayor injerencia en la gestión del hospital asumiendo la responsabilidad de guiar la institución hospitalaria. La función del médico es observar, examinar y clasificar entregándole al "especialista” un "poder-saber" que antes poseían los sacerdotes, y esto se justifica en el avance de las ciencias y sobre todo por la institucionalidad que le entrega el estado a los servicios que ofrece a la ciudadanía. El médico en su praxis profesional lo que hace es examinar una y otra vez, de forma cada vez más rápida y planificada, y además utiliza la mayor cantidad del espacio hospitalario posible, en otras palabras, el médico tiene por fin utilizar el espacio y tiempo de una institución que se sustenta en su función, tal como se comprueba con el siguiente análisis desarrollado por Michel Foucault:

La inspección de otro tiempo, discontinua y rápida, se ha trasformado en una observación regular que pone al enfermo en situación de examen casi perpetuo. Con dos consecuencias: en la jerarquía interna, el médico, elemento hasta ahora externo, comienza a adquirir preeminencia sobre el personal religioso, y se empieza a confiársele un papel determinado pero subordinado en la técnica del examen. Aparece entonces la categoría del "enfermero (2002, p. 113).

Ahora bien, la clasificación del enfermero, como el médico que atiende al enfermo supone por definición que el "especialista" utilice un espacio físico, al igual que en la cárcel, tal como se apreció anteriormente, el espacio físico es el hospital: 
el hospital-edificio es en sí mismo un 'instrumento de acción médica', en el cual los enfermos están apropiadamente separados a fin de facilitar su observación minuciosa y así ajustar mejor los cuidados; la forma de las construcciones debe tanto impedir los contagios por esta metódica separación de los enfermos, como facilitar las prácticas de permanente vigilancia y registro del avance de la enfermedad. (Pineda, 2008, s/p)

Ahora bien, en virtud de comprender la relevancia de la institución hospitalaria y su acción "disciplinadora" es que Foucault sostiene la constante dialéctica asumida por el médico en el quehacer profesional: en el hospital, el médico confronta su formación profesional con las patologías de otro sujeto denominado paciente, en primer orden, valorando, que consiste en el examen físico y psicológico del enfermo, luego clasificando, que consiste en la recolección de datos previos del paciente y por último, diagnosticando la enfermedad. Como se puede apreciar, la disciplina médica se sostiene en la constante examinación de otro sujeto, en el mayor ámbito posible, primero el cuerpo y conciencia, para luego continuar con su análisis histórico, hasta llegar a un fin que es el resultado del proceso y esto es el diagnóstico, que es la "sentencia" de un problema, en otras palabras, es el resultado de un caso investigativo y que es el médico-juez el que sentencia un diagnóstico.

En cuanto al hospital mismo, que era ante todo un lugar de asistencia, va a convertirse en lugar de formación y de confrontación de los conocimientos: inversión de las relaciones de poder y constitución de un saber. El hospital bien 'disciplinado' constituirá el lugar adecuado de la 'disciplina' médica; ésta podrá entonces perder su carácter textual, y tomar sus referencias menos en la tradición de los autores decisivos que en un dominio de objetos perpetuamente ofrecidos al examen (Foucault, 2002, p. 113).

La tercera institución que se analizará a continuación es la escuela, en ella se expresa la relación entre el profesor y el estudiante. Michel Foucault al igual que con las instituciones antes mencionadas (cárcel y hospital) establece que esta última posee una estructura rígida que se 
sustenta en un juego de poder que hace posible su mantención histórica. En la escuela se centra la formación intelectual de los jóvenes y ella es obligatoria por parte del estado para cumplir el fin de estandarización de una sociedad en particular; en la escuela, además, se puede apreciar que el examen, realizado por parte de los docentes, cumple un rol fundamental en la praxis de la escuela, donde el rol del docente se valida en la examinación de contenidos y que es la enseñanza que deben poseer los estudiantes.

la escuela pasa a ser una especie de aparato de examen ininterrumpido que acompaña en toda su longitud la operación de enseñanza. Se tratará en ella cada vez menos de esos torneos en los que los alumnos confrontaban sus fuerzas y cada vez maś de una comparación perpetua de cada cual con todos, que permite a la vez medir y sancionar (Foucault, 2002, p. 114).

Los jóvenes al "educarse" compiten unos con otros con el fin de sobresalir en una sociedad que promueve lo equilibrado, lo parecido, lo estándar, por lo tanto, la competencia por "saber más" no es más que adquirir mayor memorización entre pares con el fin de apartarse socialmente y obtener un resultado mayor que el de otros estudiantes. La educación por tanto promueve una sociedad competitiva y es el mayor productor de equidad intelectual de la sociedad, es decir, igualdad en contenidos mínimos para que sean memorizados por los estudiantes. En la escuela se vive la educación, es la institución encargada para ello y es aquí donde el docente juega un rol fundamental, en la escuela el profesor es dueño del conocimiento y dudar de ello es cuestionar el poder de la institución misma.

en la escuela, (se) crea un verdadero y constante intercambio de saberes: garantiza el paso de los conocimientos del maestro al discípulo, pero toma del discípulo un saber reservado y destinado al maestro. La escuela pasa a ser el lugar de elaboración de la pedagogía (...) la época de la escuela "examinatoria" ha marcado el comienzo de una pedagogía que funciona como ciencia (Foucault, 2002, p. 114). 
En la escuela surgen las políticas de escolarización, las cuales en su carácter fragmentario -debido a su intersección con el aspecto vital existencial, es decir, en cuanto tangencial al poder biopolítico que en la escuela como institución adquiere- permiten diversas dinámicas y por ende, tensiones. En este sentido, una lectura biopolítica de la educación, implicaría un análisis paralelo del concepto de educación en la actualidad y al mismo tiempo, un estudio de aquella educación que no se inscribe en su panorama general en virtud de su tangencialidad, es decir su carácter limítrofe: la educación urbana de extrema pobreza.

Referir, entonces, a la educación en relación con la biopolítica significa poner de relieve las vinculaciones del poder con las vidas de quienes habitan en esos espacios urbanos en los que, por sus condiciones ambientales, se hace difícil vivir; territorios, según Davis, 'hiperdegradados' (Burllaile y Orlando, 2010, s/p).

Ahora bien, la escuela es el lugar de la confrontación teórica de la formación profesional y de la práctica con los estudiantes, donde este último al ser examinado le entrega al profesor su dimensión profesional creando a su vez un estatuto epistemológico que define el lenguaje propio de la pedagogía. En otras palabras, el profesor construye la ciencia pedagógica en la medida en que él es un constante investigador que examina su objeto de estudio, que en este caso son los estudiantes, definiendo y planificando propuestas pedagógicas para obtener los resultados que se propone o le exigen realizar. El profesor es el guía de un proceso que muchas veces no tiene el fin altruista de enseñar por amor a los otros, sino todo lo contrario, el educar desde la escuela "examinatoria" e internalizadora de la norma es enseñar, evaluar y corregir constantemente las operatorias del cuerpo para su disciplinamiento

El sometimiento a 'observación' prolonga naturalmente una justicia' invadida por los métodos disciplinarios y los procedimientos de examen. ¿Puede extrañar que la prisión celular con sus cronologías ritmadas, su trabajo obligatorio, sus instancias de vigilancia y de notación, con sus maestros de normalidad, que relevan y multiplican las funciones del juez, se haya convertido en el instrumento moderno de la penalidad? ¿Puede extrañar que 
la prisión se asemeje a las fábricas, a las escuelas, a los cuarteles, a los hospitales, todos los cuales se asemejan a las prisiones? (Foucault, 2002, p. 138).

En definitiva, las instituciones se construyen a partir del régimen de disciplina, como un aparato de regulación social y política, docilizando los Cuerpos en post de un control individualizado $y$, a nivel macro, social. Lo que les permite a tales instituciones este específico modo de operar es precisamente el examen, sea clasificador o diagnóstico como dispositivos de disciplinamiento de los cuerpos.

Es hora de cuestionar las instituciones y visibilizar sus propósitos para transformar la sociedad en pos de la búsqueda de un quehacer autentico del individuo y no determinado.

\section{Referencias:}

Burllaile, M. y Orlando, G. (2010). "Biopolítica y tiempo escolar. La consistencia del tiempo en escuelas ubicadas en contextos de extrema pobreza urbana". En: Cassigoli, I. Sobarzo, M. (eds). Biopolíticas del Sur. Chile: Editorial Arcis.

Castro, E, (2004). Diccionario de Michel Foucault, Un recorrido alfabético por sus temas, conceptos y autores. Argentina: Universidad Nacional de Quilmes.

Castro, R. (2008). Foucault y el cuidado de la libertad. Ética para un rostro de arena. Chile: Editorial LOM.

Descartes, R. (1995). Discurso del método. España: Editorial Alianza.

Foucault, M. (1977). Historia de la sexualidad 1: la voluntad de poder. México: Editorial Siglo XXI.

Foucault, M. (1986). La voluntad de saber. Colombia: Editorial Siglo XXI.

Foucault, M. (2002). Vigilar y castigar. Traducción de: Aurelio Garzón del Camino. Argentina: Editorial Siglo XXI.

Foucault, M. (2006). Defender la sociedad. Traducción de Horacio Pons. Argentina: Fondo de Cultura Económica. 
Huber, P. (1968). Michel Foucault: Más allá del Estructuralismo y la Hermenéutica. Traducción de Corina de Iturbe. México: Universidad Autónoma de México.

Orrego, J. (2001). Apuntes de clases. Santiago de Chile. Extraído desde: http://u.jimdo.com/www39/o/s73705fd50dada36d/download/m068806c94d97925a/13120 89744/Los+Bienes.pdf?px-hash=b9248af561bc3d404b49951db097eb270b3c489b\&pxtime $=1373293796$.

Pineda, F. (2008). "El cuadro, la fábrica y el hospital: la producción del espacio en la época clásica". Revista Eidos [online]. n.8 [cited 2013-10-27], ISSN:2011-7477. Disponible en: http://www.scielo.org.co/scielo.php?script=sci_arttext\&pid=S1692$88572008000100005 \& \operatorname{lng}=e n \& n r m=i s o$.

Revel, J. (2008). El vocabulario de Foucault. Traducción de Víctor Golsdtein. Argentina: Atuel. 